MATHEMATICS OF COMPUTATION

Volume 71, Number 239, Pages 1323-1328

S 0025-5718(01)01374-6

Article electronically published on November 28, 2001

\title{
TEN CONSECUTIVE PRIMES IN ARITHMETIC PROGRESSION
}

\author{
H. DUBNER, T. FORBES, N. LYGEROS, M. MIZONY, H. NELSON, \\ AND P. ZIMMERMANN
}

\begin{abstract}
In 1967 the first set of 6 consecutive primes in arithmetic progression was found. In 1995 the first set of 7 consecutive primes in arithmetic progression was found. Between November, 1997 and March, 1998, we succeeded in finding sets of 8,9 and 10 consecutive primes in arithmetic progression. This was made possible because of the increase in computer capability and availability, and the ability to obtain computational help via the Internet. Although it is conjectured that there exist arbitrarily long sequences of consecutive primes in arithmetic progression, it is very likely that 10 primes will remain the record for a long time.
\end{abstract}

\section{BACKGROUND}

It is conjectured that there exist arbitrarily long sequences of consecutive primes in arithmetic progression (AP) [4]. In 1967, Lander and Parkin [5] reported finding the first and smallest sequence of 6 consecutive primes in AP, where the starting prime is 121174811 and the common difference is 30. Since then many other sets of 6 such primes have been found, as well as other sets of 6 primes with larger common differences [8].

In 1995, Dubner and Nelson found 7 consecutive primes in AP with the primes having 97 digits with a common difference of 210 [3]. The 7 consecutive primes imply that there is a prescribed set of at least 1254 numbers between the first and last prime that must be composite. This simultaneous requirement for having 7 primes in AP and also a large set of composite numbers was the reason for the difficulty in finding 7 consecutive primes in AP. The search was made practical by extending a method of Nelson for assuring that a given set of numbers would be composite [7]. The search took about 70 computer-days using PC486/66 computers. Finding 8 consecutive primes in AP would be expected to take about 20 times longer so there was no attempt to continue the project at that time.

In October 1997, Lygeros, Mizony and Zimmermann analyzed the problem of finding sets of consecutive primes such that $p(n) / n$ all have the same remainder. They discovered that the problem reduced to finding $k$ consecutive primes in arithmetic progression with certain size limitations [1] [6]. Applying the methods developed for finding 7 consecutive primes in AP, they found a solution to their problem in a matter of days.

It became apparent that the increase in computer capability and availability now made it reasonable to search for 8 consecutive primes in AP. Using about 12

Received by the editor June 22, 1998 and, in revised form, October 10, 2000.

2000 Mathematics Subject Classification. Primary 11N13.

Key words and phrases. Consecutive primes, arithmetic progression. 
computers available to the authors, 8 primes were found on November 3, 1997 after ten days which was about the expected search time.

A search for 9 primes could take an average of 6000 computer-days (Pentium/166 equivalent). We posted notices on several newsgroups and forums on the Internet and soon had about 100 helpers using about 200 computers and found 9 consecutive primes in AP on January 15, 1998.

We estimated that the expected search time for finding 10 primes would be 300 computer-years. Although this is quite a large amount of computer time the search was considered feasible since there are groups on the Internet which can have several thousand computers work on a problem simultaneously.

\section{Density OF CONSECUTIVE PRIMES}

In this section we present a heuristic analysis of the problem leading to the strategy used in the search, and in particular the choice of $N_{m}$ given by (2.5). Throughout we assume statistical independence. Although this is not strictly correct, it is adequate for our purpose. Much of what follows appeared in the 7-prime paper [3], but we include it here for the convenience of the reader.

The approximate probability of finding $k$ primes near a number $N$ is

$$
P(k) \approx\left(\frac{1}{\log N}\right)^{k} .
$$

The common difference for $k$ primes in AP must include as factors all the primes less than or equal to $k$. For the minimum common differences $d$, the minimum number, $r$, of required composites is,

$$
r=(k-1) \cdot(d-1), \begin{cases}d=30 & \text { for } k=5,6, \\ d=210 & \text { for } k=7,8,9,10, \\ d=2310 & \text { for } k=11,12, \\ d=30030 & \text { for } k=13,14,15,16,\end{cases}
$$

and the approximate probability of finding $r$ composites near $N$ is

$$
C(k) \approx\left(1-\frac{1}{\log N}\right)^{r} .
$$

Thus, for a random search near $N$, the approximate probability of finding $k$ consecutive primes in AP is the product of equations (2.1) and (2.3),

$$
Q(k) \approx\left(\frac{1}{\log N}\right)^{k} \cdot\left(1-\frac{1}{\log N}\right)^{r} .
$$

For each $k$ we can calculate the value of $N$ that optimizes the probability $Q(k)$,

$$
\log N_{m}=1+\frac{r}{k} \text {. }
$$

Near such an $N_{m}$ is where the density of $k$ consecutive primes in AP should be at a maximum. Table 1 summarizes these results up to $k=13$.

The product of $Q(k)$ and $N_{m}$ gives the expected number of $k$ sets in the range $N_{m}$ to $2 N_{m}$. For $k=7$ this product is about $10^{59}$, and for $k=13$ this product is about $10^{11976}$. This product grows faster than exponentially as $k$ increases 
Table 1. Density of Consecutive Primes in Arithmetic Progression

\begin{tabular}{rrrcrl}
$\begin{array}{c}\text { number of } \\
\text { consecutive } \\
\text { primes }\end{array}$ & $\begin{array}{c}\text { number } \\
\text { of } \\
\text { composites }\end{array}$ & $\begin{array}{c}\text { digits } \\
\text { for } \max \\
\text { density }\end{array}$ & \multicolumn{2}{c}{ approximate probabilities } \\
\hline 5 & 116 & 11 & $9 * 10^{-8}$ & $9 * 10^{-3}$ & $9 * 10^{-10}$ \\
6 & 145 & 11 & $4 * 10^{-9}$ & $3 * 10^{-3}$ & $9 * 10^{-11}$ \\
7 & 1254 & 78 & $1 * 10^{-16}$ & $1 * 10^{-3}$ & $2 * 10^{-19}$ \\
8 & 1463 & 80 & $8 * 10^{-19}$ & $3 * 10^{-4}$ & $3 * 10^{-22}$ \\
9 & 1672 & 81 & $4 * 10^{-21}$ & $1 * 10^{-4}$ & $5 * 10^{-25}$ \\
10 & 1881 & 82 & $2 * 10^{-23}$ & $4 * 10^{-5}$ & $8 * 10^{-28}$ \\
11 & 23090 & 910 & $5 * 10^{-37}$ & $1 * 10^{-5}$ & $5 * 10^{-42}$ \\
12 & 25399 & 920 & $1 * 10^{-40}$ & $6 * 10^{-6}$ & $7 * 10^{-47}$ \\
13 & 360348 & 12040 & $2 * 10^{-58}$ & $2 * 10^{-6}$ & $4 * 10^{-64}$
\end{tabular}

which heuristically lends support to the conjecture that there are arbitrarily long sequences of consecutive primes in AP.

\section{SEARCH TIME}

The method for finding 10 consecutive primes in AP is the same as that described in the 7-prime paper. 10 primes in AP with a common difference of 210 must be found simultaneously with a prescribed set of 1881 composite numbers between the first and last prime. Let

$$
\begin{aligned}
& y_{n}=x+m \cdot n, \quad n=0,1,2,3, \ldots \quad \text { where } \\
& m=\prod_{1}^{j} p_{i}, \quad p_{i}=i \text {-th prime. }
\end{aligned}
$$

The value of $x$ is determined by solving a system of $j$ congruences which guarantees that most of the intermediate numbers are composite. Each $y_{n}$ is a potentially good starting point for finding $k$ consecutive primes in AP. The search is speeded up considerably by using appropriate sieving methods on $n$.

From the 7-prime paper we can compute the expected number, $n_{t}$, of $n$ 's that must be tested in order to find a $k$ prime solution.

$$
\begin{aligned}
\frac{1}{n_{t}} & \approx\left(\frac{\log p_{j}}{.562 \cdot \log N}\right)^{k} \cdot\left(1-\frac{\log p_{j}}{.562 \cdot \log N}\right)^{s}, \quad \text { where } \\
k & =\text { number of primes in AP, } \\
s & =\text { number of remaining undetermined "composites." }
\end{aligned}
$$

Table 2 shows the expected search times for 8,9 and 10 primes where $N$ is about 93 digits, corresponding to $j=44$ with $p_{44}=193$. The times are somewhat better than the original estimates since there were continuing software improvements. The test rate per hour is, of course, very machine dependent. We show the rates for a Pentium/166 since this appeared to represent the average computer that was used. We assume that the computer was running the latest version of the software. Almost all the computers used had test rates between half and twice the rates shown. 
TABle 2. Estimated Search times for Consecutive Primes in AP (Pentium/166)

\begin{tabular}{rrrrr}
$\begin{array}{c}\text { primes } \\
\text { in AP } \\
k\end{array}$ & $\begin{array}{c}\text { remaining } \\
\text { composites } \\
s\end{array}$ & $\begin{array}{c}n \text { 's to be } \\
\text { tested } \\
n_{t}\end{array}$ & $\begin{array}{r}\text { test rate } \\
\text { per hour }\end{array}$ & $\begin{array}{c}\text { expected } \\
\text { test time } \\
\text { computer-years }\end{array}$ \\
\hline 8 & 66 & $1.3 * 10^{12}$ & $500 * 10^{6}$ & 0.29 \\
9 & 85 & $67 * 10^{12}$ & $1200 * 10^{6}$ & 6.40 \\
10 & 100 & $3000 * 10^{12}$ & $1800 * 10^{6}$ & 190.
\end{tabular}

\section{Results}

Two different programs were used to search for both 9 and 10 primes: the first was written in $\mathrm{C}$ and assembler to run on IBM compatible PC's under Windows; the second was based on the GMP library and runs on Unix work stations and PC's under Linux. Our original estimate for the expected search time to find 9 primes was 6000 computer-days. We recruited about 100 helpers using about 200 computers. After about two months, on January 15, 1998, 9 consecutive primes in AP were found after using about twice the total CPU time that was expected.

We estimated that the expected search time for finding 10 consecutive primes in AP was 300 computer-years. Despite the magnitude of the required computer time, the project was considered feasible since there are groups on the Internet that can access and monitor several thousands of computers automatically. We contacted one such organization to arrange such an approach. In preparation, we finalized the 10-prime programs and asked the 9-prime helpers to assist in some preliminary testing. Completely unexpectedly, on March 2, 1998, 10 consecutive primes in AP were found during the test phase after only $3 \%$ of the estimated total CPU time was used.

The 8-, 9- and 10-prime solutions are shown in Table 3. The probable primes were verified by using the APRT-CLE program in UBASIC, and just to be sure, they were certified again by the ECPP program of François Morain. All of these solutions fulfill the size requirements that suggested the restarting of this project. We also found an additional solution for 9 primes, 28 additional solutions for 8 primes and hundreds of solutions for 7 primes.

\section{FUTURE WORK}

Table 1 shows that if 11 consecutive primes in AP were found, the size of the primes would be about 900 digits with a prescribed set of 23,090 composite numbers. The search would take at least $10^{12}$ times longer than the search for 10-primes. It appears that the 10-prime record will stand for a long time. Of course, there is always hope that new theory will be developed which would make an 11-prime search feasible.

Extending this work for less than 11 primes could involve increasing the common difference, or increasing or decreasing the size of the primes. A particularly challenging problem that may be possible to solve with existing technology is to find the smallest 7 consecutive primes in AP. The smallest such set that is known consists of 37 digit primes and the solution is shown in Table 3. The largest such primes have 188 digits each. 
Table 3. Consecutive Primes in Arithmetic Progression

$$
P_{1}=\text { first prime }=x+m * n
$$

Common difference $=210$

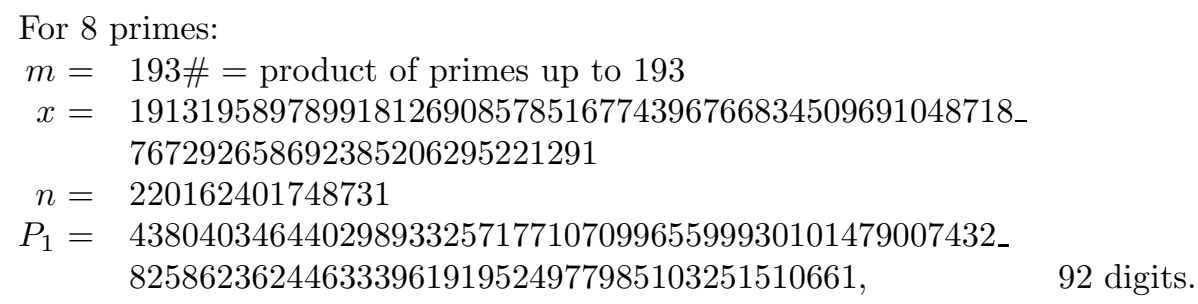

For 9 primes:

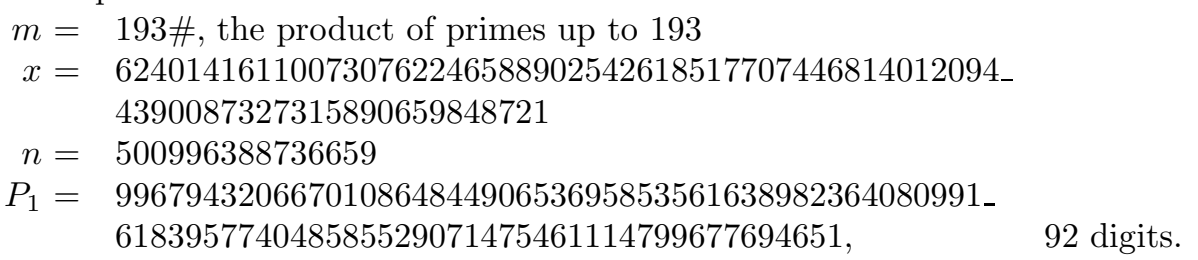

For 10 primes:

$m=193 \#$, the product of primes up to 193

$x=545382416838875826681897035901106590578659347646048$ _ 73840781923513421103495579

$n=507618446770482$

$P_{1}=10099697246971424763778665558796984032950932468919$ _ 0041803603417758904341703348882159067229719, 93 digits.

For smallest known 7 primes:

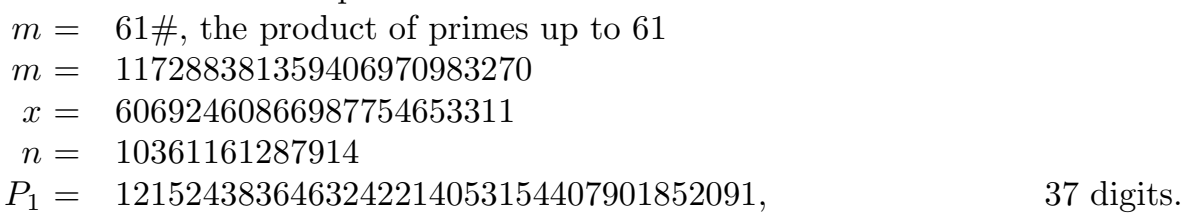

\section{ACKNOWLEDGMENTS}

We would like to thank Torbjörn Granlund for making available the free, portable and efficient GMP library on which the Unix search program was based, and also for suggesting many improvements in that program. We also wish to congratulate Manfred Toplic of Klagenfort, Austria for finding both the 9-prime and 10-prime solutions.

\section{REFERENCES}

1. M. Balazard \& A. Vavoda, Division euclidienne, Pour la Science 239, septembre 1997.

2. H. Dubner, The Size of Prime Factorials, J. Recreational Math. 19(1), 1987, pp. 743-749.

3. H. Dubner \& H. Nelson, Seven Consecutive Primes in Arithmetic Progression, Math. Comp. 66, (1997), pp. 1743-1749. MR 98a:11122 
4. R. K. Guy, Unsolved Problems in Number Theory, 2nd ed. Springer-Verlag, 1994, pp. 15-18. MR 96e:11002

5. L. J. Lander \& T. R. Parkin, Consecutive Primes in Arithmetic Progression, Math. Comp. 21(1967), p. 489. MR 37:6237

6. N. Lygeros \& M. Mizony \& P. Zimmermann, Sur la division euclidienne d'un nombre premier par son rang, Journée de Mathématiques Effectives, Départment de Mathématiques de l'Université Jean Monnet, Nov. 1998.

7. H. L. Nelson, There is a Better Sequence, J. Recreational Math. 8(1), 1975, pp. 39-43.

8. S. Weintraub, Consecutive Primes in Arithmetic Progression, J. Recreational Math. 25(3), 1993, pp. 169-171.

449 Beverly Road, Ridgewood, New Jersey 07450

E-mail address: hdubner1@compuserve.com

22 St. Albans Road, Kingston upon Thames, Surrey, KT2 5HQ England

E-mail address: tonyforbes@ltkz.demon.co.uk

Institut Girard, Cnr Upres-A 502B, Université Lyon I 43 Bd Du 11 Novembre 1918, 69622 Villeurbanne Cedex, France

E-mail address: lygeros@desargues.univ-lyon1.fr

Institut Girard, Cnr Upres-A 502B, Université Lyon I 43 Bd Du 11 Novembre 1918, 69622 Villeurbanne Cedex, France

E-mail address: mizony@desargues.univ-lyon1.fr

4259 Emory Way, Livermore, California 94550

E-mail address: hlnel@flash.net

Inria Lorraine, Technopole de Nancy-Brabois, 615 Rue Du Jardin Botanique Bp 101, F-54600 Villers-Lès-NANCy, France

E-mail address: zimmerma@loria.fr 